\title{
Helicity-independent optical pumping of nuclear magnetization in bulk CdTe
}

\author{
Wei Dong, ${ }^{1}$ Bo Li, ${ }^{1}$ Qiang Zhang, ${ }^{2}$ Maria C. Tamargo, ${ }^{2}$ and Carlos A. Meriles ${ }^{1}$ \\ ${ }^{1}$ Department of Physics, The City College of New York, CUNY, 138th Street and Convent Avenue, New York, New York 10031, USA \\ ${ }^{2}$ Department of Chemistry, The City College of New York, CUNY, 138th Street and Convent Avenue, New York, New York 10031, USA
}

(Received 27 April 2009; published 30 July 2009)

\begin{abstract}
We report on the observation of optically pumped nuclear spin polarization in samples of CdTe at high magnetic field using ${ }^{125} \mathrm{Te}$ NMR. We find strong enhancement both in bulk material as well as in a molecular beam epitaxy grown, $2 \mu$ thick epilayer. Contrary to prior results, however, we observe that the magnitude and orientation of the nuclear spin alignment is independent of laser helicity. Further, we find that after its onset at sub-bandgap energies, the nuclear polarization rapidly increases to reach $\sim 3 \%$ and remains virtually unchanged as higher energies are scanned. Comparison with additional data from a semi-insulating GaAs crystal points to a polarization mechanism different from that typically dominant, possibly driven by surface spindependent recombination.
\end{abstract}

DOI: 10.1103/PhysRevB.80.045211

PACS number(s): 71.55.Gs, 76.60.-k, 82.56.Na

The interplay between electron and nuclear spins in semiconductors and the optical enhancement of nuclear polarization are topics of continuing interest due to the promise of spin-based quantum computation and sensitivity-enhanced magnetic resonance. While, in general, most recent studies have focused on "optically pumped" spins in confined structures, a string of observations point to a still incomplete understanding of the dynamics of nuclear polarization in bulk systems. For example, measurements of the nuclear polarization in GaAs derived from the asymmetries of quadrupolarsplit NMR lines seem to suggest that both localized and delocalized electrons collaborate in the optical pumping (OP) process. ${ }^{1}$ And while the Fermi contact contribution to the hyperfine coupling was assumed dominant in direct semiconductors, the role played by the dipolar component still remains controversial. ${ }^{2,3}$ Another example is the recent work of Hayes and collaborators ${ }^{4,5}$ who have shed light on some of the intricacies in the electro-optical processes leading to nuclear OP in bulk GaAs.

The results we present herein focus on the dynamics of nuclear polarization in bulk CdTe. We use ${ }^{125} \mathrm{Te}$ NMR to directly monitor the optically enhanced nuclear polarization at high magnetic field both in a single crystal and in a molecular beam epitaxy (MBE)-grown CdTe epilayer. We find an intriguing dependence of the induced nuclear polarization with photon energy and helicity that cannot be explained within the traditional theoretical framework, thus pointing to alternate polarization pathways. Although it is not possible at this time to rule out other mechanisms, our observations seem consistent with spin-dependent recombination via surface shallow donors.

To more clearly introduce our results we recall that in direct-bandgap semiconductors, optical pumping of nuclear spins is typically described as a transfer process, in which the angular momentum of circularly polarized light cascades down to electrons and then to nuclear spins. The first step is made possible by the optical selection rules present in this type of materials: ${ }^{6}$ With the $z$ axis representing the direction of illumination, the corresponding projection of the electron spin immediately after light excitation is predicted to have a value $S_{z}= \pm 0.25$, depending on the helicity of the beam.
Under steady-state conditions and assuming the Faraday geometry (i.e., illumination parallel to the external magnetic field), the resulting, quasiequilibrium spin magnetization is given by $^{7}$

$$
S_{e q}=S_{z} /\left(1+\tau / T_{1 e}\right)+S_{0} /\left(1+T_{1 e} / \tau\right),
$$

where $T_{1 e}$ and $\tau$ indicate the electron spin relaxation and recombination times, respectively. $S_{0}=-(1 / 2) \tanh \left(g^{*} \mu_{B} B_{0} /\right.$ $\left.2 k_{B} T\right)$ is the thermal spin alignment of electrons with $g$ factor $g^{*}$ in a magnetic field $B_{0}$ at temperature $T ; \mu_{B}$ and $k_{B}$ denote as usual the Bohr magneton and Boltzmann constant.

The transfer of angular momentum to the nuclear spin system takes place via the hyperfine interaction between nuclei and conduction electrons; holes, on the other hand, typically play a less important role because the $\pi$-like character of the hole wave function guarantees a negligible density at the nuclear site (and thus a strongly reduced hyperfine coupling). Within this framework, enhancement of nuclear magnetization is observed when the electron-nuclear relaxation time $T_{1 H}$ is much shorter than the nuclear spin-lattice time $T_{1 L}$, and the steady-state electron polarization differs from the thermal value. If we ignore inhomogeneities in the spatial distribution, the predicted quasistationary value of nuclear polarization is given by $^{8}$

$$
I_{O P}=I_{0}+f \frac{I(I+1)}{S(S+1)} \frac{1}{\left(1+\tau / T_{1 e}\right)}\left(S_{z}-S_{0}\right),
$$

with $I_{0}$ denoting the average nuclear spin projection along the field direction in thermal equilibrium and $f \equiv 1 /(1$ $\left.+T_{1 H} / T_{1 L}\right)$ indicating a "leaking factor" due to nuclear spin relaxation with the lattice.

To directly monitor the optically enhanced nuclear polarization in our samples, we relied on a setup similar to that described previously. ${ }^{9}$ High-field, variable-temperature experiments were carried out using a 9.4 T NMR magnet and a continuous flow cryostat with optical access. The sample was in good thermal contact with a sapphire wafer to facilitate heat dissipation, and a Cernox sensor (additional to the builtin, cryostat resistance temperature detector (RTD)) was placed in its proximity to provide more reliable temperature 
readout. Laser illumination was, as usual, parallel to the direction of the magnetic field (and perpendicular to the sample surface); the addition of an optical expander allowed us to vary the beam diameter from $\sim 3$ to $0.5 \mathrm{~mm}$. To excite and detect nuclear spins we used a homemade, two-channel probe head; cryogenic capacitors allowed us to tune and match the rf circuit down to $4 \mathrm{~K}$ and over a broad range of frequencies. We studied three samples: the first one was a semi-insulating CdTe single crystal from University Wafers (resistivity $>10^{9} \Omega \mathrm{cm}$, surface orientation [111]). The second one was a $2 \mu$ thick, MBE-grown CdTe epilayer (surface orientation [100]) on a GaAs substrate; a thin $\mathrm{ZnSe}$ layer $(1 \mathrm{~nm})$ was intercalated between the CdTe film and the substrate to improve the film quality. ${ }^{10}$ Finally, we used a semi-insulating GaAs single crystal as a control (350 $\mu$ thick, surface orientation [100], resistivity greater than $10^{7} \Omega \mathrm{cm}$, American Crystal Technologies). Samples were used "as received" (although in subsequent experiments we did clean the surface of the CdTe crystal using methanol with no apparent change).

Detection of nuclear polarization was preceded by the known protocol $S A T-\tau_{L^{-}} \tau_{D^{-}} \pi / 2$ where $\tau_{L}$ and $\tau_{D}$ indicate illumination and dark intervals, respectively, and $\pi / 2$ represents the excitation rf pulse. Saturation (SAT) prior to illumination was carried out via a string of resonant $\pi / 2$ pulses. ${ }^{125}$ Te NMR spectra for both the CdTe single crystal and film at sub-bandgap energies are shown in Fig. 1. The beam diameter was $\sim 1 \mathrm{~mm}$ and the laser power was $300 \mathrm{~mW}$. Consistent with the long relaxation times found in CdTe (of order $\sim 10 \mathrm{~min}$ at room temperature and exceeding several hours below $80 \mathrm{~K}$ ), no signal was observed in the dark. On the other hand, strong enhancements that do not depend on the beam polarization were found in both samples when the laser was on. Notably, the signal amplitude in the film was almost identical to that observed in the crystal implying that polarized nuclei lie within less than $2 \mu$ from the surface. Using the room-temperature signal from the $5 \times 5 \times 0.35 \mathrm{~mm}^{3}$ CdTe crystal as a reference and assuming an effective volume of illumination of $\sim 1 \times 1 \times 2 \times 10^{-3} \mathrm{~mm}^{3}$, we estimate a nuclear polarization of order $3 \%$.

In spite of several attempts, practical limitations did not allow us to determine the sign of the induced alignment relative to thermal polarization: in the absence of illumination, the above-mentioned nuclear relaxation times make comparison after OP exceedingly difficult. On the other hand, comparison with the signal from partially relaxed nuclear spins immediately after the system cool down but before optical excitation was prevented by our failure to detect crystal nuclei in a single scan. (Note in this regard that given the relative dilution of ${ }^{125} \mathrm{Te}$ and small crystal size several scans are necessary to attain reasonable signal-to-noise ratio at room temperature).

The response to light helicity observed in our CdTe samples differs from that seen in our control GaAs crystal where, in agreement to well-known results, signal inversion takes place when passing from right-to-left circularly polarized illumination. More importantly, our results differ from those reported previously in a CdTe crystal subject to similar conditions: ${ }^{11}$ Though no inversion was present, this latter case showed a significant change between signals induced by

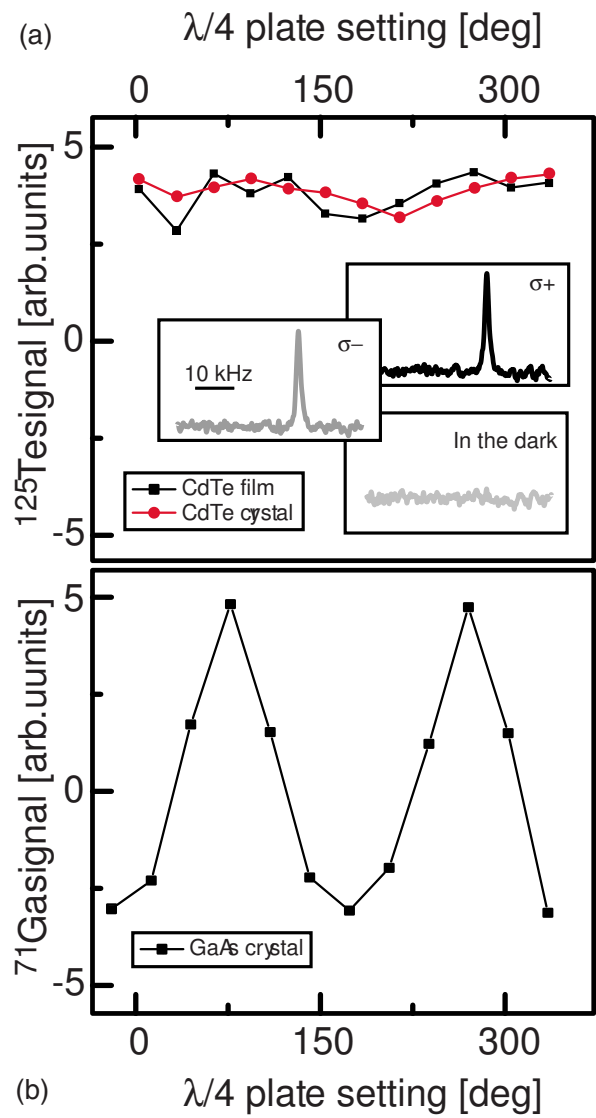

FIG. 1. (Color online) (a) ${ }^{125} \mathrm{Te}$ optically enhanced signal as a function of light helicity (expressed via the quarter-wave plate setting). Squares $(\boldsymbol{\square})$ and circles $(\bigcirc)$ indicate the NMR signal from film and crystal, respectively. Note that the relative amplitude is close to unity implying that the signal originates from superficial nuclei. The inserts show actual (crystal) spectra in the absence of illumination and for excitation light of opposite helicity (FWHM $\sim 2 \mathrm{kHz}$ ). The magnetic field is $9.4 \mathrm{~T}$, the temperature is $5 \mathrm{~K}$, the laser power $300 \mathrm{~mW}$, and the beam diameter $1-2 \mathrm{~mm}$. The illumination wavelength was $800 \mathrm{~nm}, \tau_{D}=10 \mathrm{~s}$ and $\tau_{L}=300 \mathrm{~s}\left(\tau_{L}\right.$ $=1200 \mathrm{~s}$ in the inserts). (b) Corresponding results in a control crystal of semi-insulating GaAs under similar experimental conditions (light excitation at $825 \mathrm{~nm}$ ). To facilitate comparison, the absolute amplitude of the ${ }^{71} \mathrm{Ga}$ NMR signal was multiplied by a factor two.

light of different helicity. Further, when plotted as a function of photon energy, the signal amplitude was observed to peak slightly below the bandgap to sharply decay to a lower plateau at higher energies (a behavior somewhat reminiscent of that observed in GaAs). ${ }^{9}$ As shown in Fig. 2, our samples exhibit a different dependence: the NMR signal does reach a maximum at sub-bandgap energies but then it remains virtually unchanged as higher photon energies are probed (note in this regard the broad energy range reaching $1.77 \mathrm{eV}$ or 700 $\mathrm{nm})$.

Given the disparities pointed above, we made every effort to ensure the absence of systematic errors in our experiments; a particular concern was inadvertent laser heating, a parameter difficult to monitor and known to have nonnegligible effects in OPNMR. ${ }^{12}$ While results in our control GaAs sample suggest that our experiments are exempt from 




FIG. 2. (Color online) (Main) ${ }^{125}$ Te NMR signal amplitude as a function of the photon energy. Squares $(\boldsymbol{\square})$ and circles $(\bigcirc)$ indicate the crystal signal for left- $\left(\sigma_{-}\right)$and right- $(\sigma+)$ circularly polarized light while upright $(\boldsymbol{\Delta})$ and inverted $(\boldsymbol{\nabla})$ triangles provide identical information for the film. Experimental parameters as listed in Fig. 1. The dashed line indicates the bandgap energy at this temperature as determined from transmission measurements in our CdTe crystal. This value is in excellent agreement with that reported in the literature. (Ref, 15) (Inset) Energy dependence for our control GaAs crystal under right circularly polarized illumination.

this problem, reassuring evidence is presented in Fig. 3: similar to observations in GaAs, we find a linear increase in the observed signal with light intensity and a typical exponential growth with illumination time. More importantly, when plotted as a function of increasing lattice temperature, we observe a fast polarization decay that renders the NMR signal virtually unobservable above $20 \mathrm{~K}$. (In passing, we note that no signal from the thin-film sample in the dark exceeded noise levels at any temperature further demonstrat-

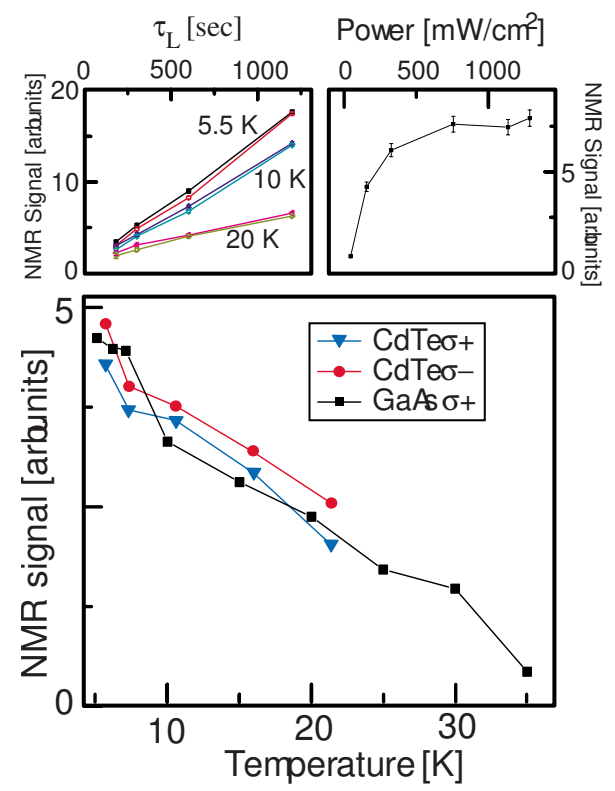

FIG. 3. (Color online) ${ }^{125}$ Te NMR signal amplitude as a function of illumination time $\tau_{L}$, laser power density, and temperature. For comparison, the last figure includes (scaled) ${ }^{71} \mathrm{Ga}$ results in our control GaAs sample. Experimental conditions as listed in Fig. 1. wavelength is $800 \mathrm{~nm}$ for CdTe and $825 \mathrm{~nm}$ for GaAs.

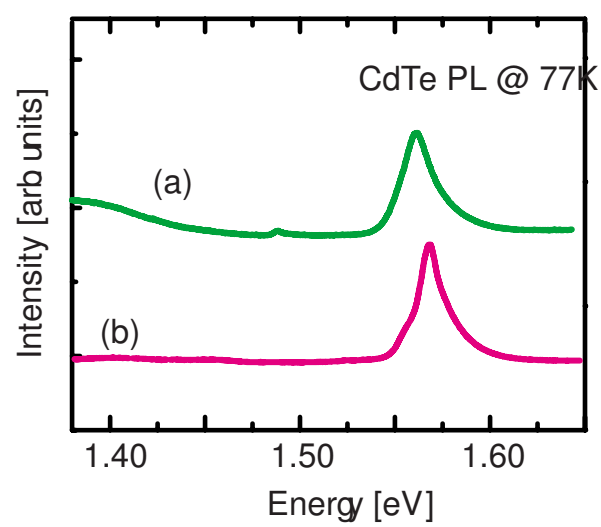

FIG. 4. (Color online) Photoluminescence spectra from (a) the CdTe crystal and (b) the CdTe film at $77 \mathrm{~K}$ and zero magnetic field. The excitation wavelength is $1.77 \mathrm{eV}(700 \mathrm{~nm})$. Observe in both cases the tail toward higher energies. Deep level emission (around $1.4 \mathrm{eV}$ ) is only present in the CdTe crystal.

ing the large magnitude of the enhancement). Remarkably, the decay rate is as fast as that found in GaAs, a somewhat unexpected observation given the longer lattice relaxation times $T_{1 L}$ of nonquadrupolar nuclear systems (all relevant spin isotopes in CdTe have spin $\frac{1}{2}$ ).

Though free excitons may participate in the process of nuclear spin polarization, ${ }^{1,9,11}$ shallow impurities and lattice defects are typically assumed to play a dominant role: ${ }^{13}$ carrier localization by lattice imperfections leads to locally enhanced hyperfine couplings and thus to efficient alignment of neighboring nuclei. Electron traps thus operate as nucleation centers of polarization, which, in turn, spreads throughout the crystal via spin "flip-flops," a mechanism described in the OP master equation by a diffusionlike term. ${ }^{14}$ As shown in Fig. 2, the observation of strong, sub-bandgap signal points to lattice imperfections driving the nuclear polarization process in our CdTe samples. ${ }^{15}$ Whether such imperfections are interstitial or substitutional impurities, vacancies, antisites, dislocations (or other types of defects) still remains to be determined. Some insight on the nature of these lattice imperfections is provided by the photoluminescence (PL) spectra shown in Fig. 4. Both CdTe samples show strong band-edge emission at $77 \mathrm{~K}$ but, unlike the crystal, the CdTe film shows virtually no deep level PL, which allows us to exclude this type of defect from those relevant to the OP process. Further, the main emission peak is slightly asymmetric toward higher energies, indicative of coexisting "bound exciton" and "free-to-bound" recombination (involving a trapped electron or hole and a free carrier).

With the intention to further characterize these nucleation centers, we also monitored the optically enhanced NMR spectra at short pumping times. The importance of this strategy has been recently demonstrated by Hayes and collaborators, who observed strong light-induced hyperfine shifts in the NMR spectrum of semi-insulating GaAs. ${ }^{5}$ Resulting from the hyperfine interaction of localized electrons with neighboring nuclei, these shifts depend on light helicity and are more notable at short illumination times (before spin diffusion spreads the nuclear polarization farther away from the trapping centers). Figure 5 shows results obtained in the 


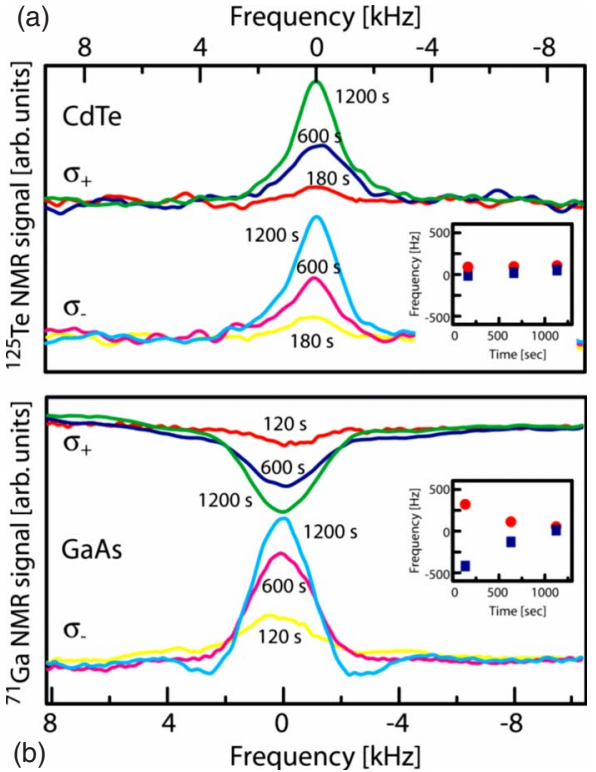

FIG. 5. (Color online) Optically pumped NMR spectra in (a) our CdTe crystal and (b) the GaAs control sample for different illumination times $\tau_{L}$. Clear, helicity-dependent Knight shifts observable at short $\tau_{L}$ in GaAs are absent in CdTe. Spectra have been shifted for clarity. The temperature is $5 \mathrm{~K}$, the laser power is $300 \mathrm{~mW}$, the ${ }^{125} \mathrm{Te}$ and ${ }^{71} \mathrm{Ga}$ central frequencies at $9.4 \mathrm{~T}$ are $\nu_{\mathrm{Te}}$ $=126.022 \mathrm{MHz}$ and $\nu_{\mathrm{Ga}}=121.965 \mathrm{MHz}$, respectively. The illumination wavelength is $800 \mathrm{~nm}$ for CdTe and $825 \mathrm{~nm}$ for GaAs.

CdTe crystal and our control GaAs sample: at $\tau_{L}=120 \mathrm{~s}$ we observe clear, helicity-dependent frequency shifts in GaAs, which progressively decay to virtually disappear at $\tau_{L}$ $=1200 \mathrm{~s}$ in qualitative agreement with the results of Ref. 5 . On the other hand, the CdTe NMR spectra do not visibly change with illumination time or light helicity. In a model where the nuclear polarization process is driven by localized electrons, this result may seem disconcerting. ${ }^{71} \mathrm{Ga}$ and ${ }^{125} \mathrm{Te}$ have comparable hyperfine couplings $A_{H}$ (of order $50 \mu \mathrm{eV})^{13,16}$ and, thus one would expect similar Knight shifts in both systems. (Note that the five-times-lower natural abundance of ${ }^{125} \mathrm{Te}$ as compared to ${ }^{71} \mathrm{Ga}$ is expected to serve only as a scaling factor, without altering the magnitude of the NMR shift).

The idea that similar hyperfine couplings lead to comparable shifts is only granted if the function describing the spatial distribution of the trapped electron is also comparable: this is because the position-dependent Knight field $B_{e}(r) \sim k_{e} A_{H} \exp \left(r / a_{0}\right)$ decreases rapidly with increasing distance $r$ to the trap. ${ }^{13}$ The scale governing this decay is $a_{0}$, the excitonic Bohr radius, found to be only $6.8 \mathrm{~nm}$ in CdTe, almost half the value in GaAs (12.0 nm). ${ }^{17}$ Keeping in mind the low natural abundance of Te pointed above, the latter has the important consequence that the Knight field affects only a small number of nuclear spins close to a lattice defect. Further, it implies that the corresponding Knight shifts are stronger in CdTe or, equivalently, that the spectral "tail" describing the distribution of lattice defects becomes broader (and thus weaker), a condition in conflict with the sensitivity of our experiment.

The behavior found in Figs. 1 and 2 is, perhaps, more intriguing: if for simplicity we ignore the nuclear thermal polarization $I_{0}$, formula (2) demands that the observed OP signal be proportional to $S_{z}-S_{0}$, i.e., proportional to the difference between the expectation values of the electron spin immediately after light excitation and in thermal equilibrium. For an effective Landé factor $g^{*} \sim 1.64$ valid in bulk CdTe, ${ }^{18}$ a magnetic field of $9.4 \mathrm{~T}$ and a temperature of $5 \mathrm{~K}$, we obtain $\left|S_{0}\right| \approx 0.38$. Therefore, the expected fractional change in the NMR signal upon reversal of the excitation beam helicity has magnitude $\left|I_{O P}^{+}-I_{O P}^{-}\right| /\left|I_{O P}^{+}+I_{O P}^{-}\right|=\left|S_{z}^{+}-S_{z}^{-}\right| /\left|2 S_{0}\right| \approx 70 \%$, in strong disagreement with our observations.

This last result points to nuclear spin-polarization pathways different from those found in other systems. The nature of this alternate mechanism is unclear at this time but we speculate that the observed behavior originates from strong spin-dependent recombination (SDR). First observed by Lepine in pure silicon, ${ }^{19}$ we recall that SDR alludes to a process in which excess electrons and holes recombine via paramagnetic impurities. When a conduction electron is captured by a paramagnetic center, the electron and center spins form intermediate singlet or triplet states. ${ }^{20}$ The former has lower energy than the latter, a condition that makes singlet states considerably more localized and, more importantly, more prone to induce exciton recombination. Further, because the total angular momentum is conserved during optical transitions, singlet electrons with a particular angular momentum projection recombine preferentially if holes have a well-defined alignment. ${ }^{3}$ The result of this process is, therefore, the selective polarization of the electron trapped at a donor.

In a SDR-dominated OPNMR experiment-where the nuclear spin polarization derives from selective recombination at shallow donors-helicity-independent signals can be expected when free carriers spin-relax before they recombine. The latter seems to be a reasonable assumption in the present case: as a matter of fact, holes are known to relax much faster than electrons in bulk, zincblende semiconductors owing to their stronger spin-orbit coupling. And while it is true that the electron spin lifetime may exceed the recombination time in certain systems, $n$-type doping (absent in our samples) is mandatory for excess carriers to spin exchange. ${ }^{21}$ More importantly, time-resolved optical measurements in CdTe epilayers very similar to the one used here ${ }^{22}$ find $T_{1 e} / \tau \sim 0.3$ at room temperature (sample labeled Y3 in Ref. 22) further confirming the idea that the nuclear polarization is helicity-insensitive simply because the optical imprint on the exciton spin is lost before it can be transferred to the nuclei. On a final note, we mention that $T_{1 e}<\tau$ is a sufficient but not necessary condition for helicity-independent nuclear signals, as other, more subtle scenarios with $T_{1 e}=\tau$ are conceivable as well.

In Lepine's paper, ${ }^{19}$ it was shown that SDR in silicon is not homogeneously distributed over the sample volume but rather restricted to the surface (where the capture cross section is enhanced via charge-induced changes in the surface potential). ${ }^{19}$ The observation that above $1.50 \mathrm{eV}$ the ${ }^{125} \mathrm{Te}$ NMR signal is insensitive to excitation energy (Fig. 2) is consistent with a surface-confined, SDR-driven OP because, once photons exceed a threshold energy, the same number of centers remains active regardless the excitation wavelength. 
As discussed above, this is not the case in semi-insulating GaAs (Fig. 2), where the amplitude of the nuclear spin signal has been shown to correlate well with the (wavelengthdependent) absorption coefficient (and therefore with the excitation volume). ${ }^{4,23}$

If confirmed, the spontaneous confinement of enhanced nuclear polarization near the surface of CdTe could find interesting applications. For example, Tycko pointed that routinely used polarization-transfer schemes could be exploited to enhance the NMR signal of an organic thin film overlaid on a semiconductor substrate. The so-called "spectroscopic barrier" precluding efficient transfer from systems of quadrupolar nuclei (e.g., GaAs) is absent in CdTe making this material a convenient choice. Note in particular that our estimate of the film nuclear polarization (3\%) presumes homogeneous distribution over the $2 \mu$ thickness; obviously, greater alignment must be present if only a thinner, superficial section is effectively pumped.

In conclusion, we have observed strong optical enhancement of nuclear polarization in bulk CdTe, both in a commercially available single crystal and in a $2 \mu$ thick, MBEgrown film. Comparison with measurements on a bulk semiinsulating GaAs sample subjected to virtually identical conditions exposes intriguing differences; chiefly among them is the fact that the nuclear polarization in CdTe is insensitive to light helicity and excitation energy (above 1.5 $\mathrm{eV}$ ). Such results are incompatible with predictions derived from the "traditional" OP theory where the resulting nuclear polarization-ultimately a byproduct of the optical selection rules of direct semiconductors-is expected to exhibit a strong change upon reversal of light helicity. While further experiments are mandatory, spin-dependent recombination via shallow paramagnetic centers near the sample surface seems a plausible nuclear polarization mechanism, particularly if, as suggested by time-resolved optical measurements, the electron-spin lifetime is shorter than the recombination time. Interestingly, surface spin-dependent recombination not only is consistent with the observed helicity and photon energy dependencies but could also be a factor behind the fast thermal decay (because the NMR signal is roughly expected to reflect the spin alignment of the paramagnetic centers driving the process).

The reasons why our results differ from those reported previously remain presently unknown to us though we recall that while the conclusion in Ref. 11 was that mobile excitons may be contributing to the OP process our results point to an impurity driven process. In this context, we note that $\mathrm{CdTe}$ surfaces have proven very sensitive to preparation conditions: ${ }^{24}$ For example, x-ray photoelectron spectroscopy studies of methanol-etched CdTe surfaces reported the preferential formation of $\mathrm{TeO}_{2}$ at the surface upon exposure to the ambient; little or no oxidation was observed, however, in specimens etched in citric acid. ${ }^{25}$ Given this scenario, several strategies seem at hand to shed light on the spatial location and, ideally, the nature of these impurities. The most straightforward seems to be the search of OPNMR signals in thinner CdTe films, but the increased density of dislocations, vacancies and other lattice defects is likely to make comparison with our present results more difficult. Alternatively, one could "passivate" the surface of a $\sim 1 \mu$, high-quality CdTe film with the subsequent growth of a "capping layer" tens of nanometers thick; note that with the selection of a transparent, wider bandgap material such as $\mathrm{ZnSe}$, differences in the observed behavior are likely to expose the role that surface impurities play in the CdTe OP process.

The authors are indebted to Jeffrey Reimer and Patrick Coles for helpful discussions. We acknowledge support from NSF through Grants No. CHE-0545461 and No. ECS0608763. C.A.M. thanks the Research Corporation for support through the Cottrell Scholar program.
${ }^{1}$ A. K. Paravastu and J. A. Reimer, Phys. Rev. B 71, 045215 (2005).

${ }^{2}$ A. Patel, O. Pasquet, J. Bharatam, E. Hughes, and C. R. Bowers, Phys. Rev. B 60, R5105 (1999).

${ }^{3}$ A. Brunetti, M. Vladimirova, D. Scalbert, H. Folliot, A. Lecorre, Phys. Rev. B 73, 121202(R) (2006).

${ }^{4}$ S. Mui, K. Ramaswamy, and S. E. Hayes, Phys. Rev. B 75, 195207 (2007).

${ }^{5}$ K. Ramaswamy, S. Mui, and S. E. Hayes, Phys. Rev. B 74, 153201 (2006).

${ }^{6}$ Optical Orientation (Modern Problems in Condensed Matter Science), edited by F. Meier and B. P. Zakharchenya (NorthHolland Physics Publishing, Amsterdam, 1984), Vol. 8.

${ }^{7}$ C. Weisbuch and C. Hermann, Phys. Rev. B 15, 816 (1977).

${ }^{8}$ A. Abragam, Principles of Nuclear Magnetism (Oxford University Press, New York, 1996)

${ }^{9}$ A. K. Paravastu, S. E. Hayes, B. Schwickert, L. N. Dinh, M. Balooch, and J. A. Reimer, Phys. Rev. B 69, 075203 (2004).

${ }^{10}$ Q. Zhan, W. Charles, B. Li, A. Shen, C. A. Meriles, and M. C. Tamargo, J. Cryst. Growth 311, 2603 (2009).
${ }^{11}$ I. J. H. Leung and C. A. Michal, Phys. Rev. B 70, 035213 (2004).

${ }^{12}$ P. J. Coles, Phys. Rev. B 78, 033201 (2008)

${ }^{13}$ D. Paget, G. Lampel, B. Sapoval, and V. I. Safarov, Phys. Rev. B 15, 5780 (1977).

${ }^{14}$ P. L. Kuhns, A. Kleinhammes, T. Schmiedel, W. G. Moulton, E. Hughes, S. Sloan, P. Chabrier, and C. R. Bowers, Phys. Rev. B 55, 7824 (1997).

${ }^{15}$ D. R. Lide, Handbook of Chemistry and Physics, 77th ed. (CRC, Boca Raton, FL, 1996), Ch. 12, pp. 12-98.

${ }^{16}$ J. Tribollet, E. Aubry, G. Karczewski, B. Sermage, F. Bernardot, C. Testelin, and M. Chamarro, Phys. Rev. B 75, 205304 (2007).

${ }^{17}$ P. Y. Yu and M. Cardona, Fundamentals of semiconductors (Springer-Verlag, Berlin, 1996).

${ }^{18}$ A. A. Sirenko, T. Ruf, M. Cardona, D. R. Yakovlev, W. Ossau, A. Waag, and G. Landwehr, Phys. Rev. B 56, 2114 (1997).

${ }^{19}$ D. J. Lepine, Phys. Rev. B 6, 436 (1972).

${ }^{20}$ C. Boehme and K. Lips, Phys. Rev. Lett. 91, 246603 (2003).

${ }^{21}$ R. I. Dzhioev, K. V. Kavokin, V. L. Korenev, M. V. Lazarev, B. Ya. Meltser, M. N. Stepanova, B. P. Zakharchenya, D. Gammon, 
and D. S. Katzer, Phys. Rev. B 66, 245204 (2002).

${ }^{22}$ P. Nahálková, P. Němec, D. Sprinzl, and E. Belas, Mater. Sci. Eng., B 126, 143 (2006).

${ }^{23}$ P. J. Coles and J. A. Reimer, Phys. Rev. B 76, 174440 (2007).
${ }^{24}$ F. Wang, A. Schwarzman, A. L. Fahrenbruch, R. Sinclair, R. H. Bube, and C. M. Stahle, J. Appl. Phys. 62, 1469 (1987).

${ }^{25}$ S. P. Kowalczyk and J. T. Cheung, J. Vac. Sci. Technol. 18, 944 (1981). 